\title{
An Unusual Cause of Ureter Rupture and Acute Abdomen
}

\author{
Kenneth S. Fink, $M D, M G A$
}

Radium implants have been used in the past to treat cervical cancer, and much has been written about the complication of ureteral stricture. ${ }^{1-6}$ Ureteral rupture is a well-documented urologic problem resulting from numerous iatrogenic and noniatrogenic causes, including ureteroscopy, ${ }^{7}$ elective pregnancy termination, ${ }^{8}$ both penetrating and blunt trauma, ${ }^{9-11}$ and urolithiasis. ${ }^{12,13}$ In reviewing the literature for complications of radium treatment and for causes of ureter rupture, no case was found that described ureter rupture following treatment with radium. To my knowledge this case is the first such reported.

A patient with an acute abdomen poses a diagnostic challenge to the physician. In approaching such a patient, it is necessary to form a broad set of differential diagnoses. This case report describes a patient who came to the emergency department with an acute abdomen and was found to have a spontaneous ureter rupture. ${ }^{14}$ She had received two treatments with radium implants for cervical cancer more than 35 years earlier, which resulted in ureter fibrosis, stricture, obstruction, and subsequent perforation.

\section{Case Report}

The patient was a 70-year-old woman with a history of coronary artery disease and irritable bowel syndrome who had had four-vessel coronary artery bypass grafts 1 year earlier and cervical cancer treated by radium implant placement 37 years and again 34 years ago. She also stated she was hospitalized for 28 days 3 years earlier for diverticulitis, but the available records merely stated that she had colitis in the past with no specific diagnosis recorded.

She came to the emergency department with a

Submitted, revised, 21 November 1997

From the Department of Family Medicine, School of Medicine, University of Washington, Seattle. Address reprint requests to Kenneth S. Fink, MD, MGA, Department of Family Medicine, UW Medical Center, Box 354775, Seattle, WA 98105. 3-day history of diffuse abdominal pain, nausea, diarrhea, and urinary frequency and urgency. Her temperature was $98.9^{\circ} \mathrm{F}$, blood pressure $130 / 86$ $\mathrm{mmHg}$, pulse 72 beats per minute, and respirations $20 / \mathrm{min}$. Her physical examination was remarkable for a soft abdomen, which was tender over the whole surface without rebound tenderness. Bowel sounds were decreased. She had bilateral costovertebral angle tenderness. Her urinalysis showed 1 to 3 white cells and 3 to 5 red cells per high-power field and was negative for nitrite and leukocyte esterase; her complete blood count was notable for a white cell count of $11,700 / \mu \mathrm{L}$ with 78 percent neutrophils. A serum chemical analysis showed blood urea nitrogen $24 \mathrm{mg} / \mathrm{dL}$ and creatinine $0.9 \mathrm{mg} / \mathrm{dL}$; other values were normal. A urine culture was not requested. Her condition was diagnosed as an atypical urinary tract infection, and ciprofloxacin, $500 \mathrm{mg}$ by mouth twice a day, was prescribed. She received 25 $\mathrm{mg}$ of meperidine before discharge.

The patient returned to the emergency department a few hours later with severe, sharp, constant pain in the left lower quadrant of her abdomen. Although she had been having some diarrhea, she had begun to have emesis, but she remained afebrile. She denied any melena, hematochezia, or hematemesis. She stated that she felt the same as when she was admitted 3 years previously for diverticulitis. Findings of the physical examination were remarkable for left costovertebral angle tenderness and diffuse abdominal tenderness greatest in the left lower quadrant with involuntary guarding but no rebound. Stool was guaiac negative. A repeat complete blood count showed a white cell count of $12,800 / \mu \mathrm{L}$ with 87 percent neutrophils. Abdominal radiographs showed no free air or any air-fluid levels.

The patient was admitted for observation with presumed diverticulitis. She tolerated oral ciprofloxacin, $500 \mathrm{mg}$ twice a day, and clindamycin, $300 \mathrm{mg}$ four times a day, required minimal pain medication, and was discharged the following 
morning to continue her oral treatment as an outpatient.

The patient returned to the clinic 2 days later with worsening pain and now vomiting. The only change found on her physical examination was increased tenderness, still localized in her left lower abdomen. Abdominal radiographs were repeated, and again the findings were normal. An abdomnal sonogram was remarkable for moderate left hydronephrosis with flow of urine into the bladder from the left ureter. She was again hospitalized and given an intravenous antibiotic combination of ampicillin and sulbactam (Unasyn), first with a 3 -g bolus and then $1.5 \mathrm{~g}$ every 6 hours. The patient did not improve after 24 hours of treatment, and a surgical consultation was requested. Abdominal computed tomograms were obtained, which showed extravasation of urine with tracking along the ureter, moderate left hydronephrosis, no stones, and opacification of portions of the distal left ureter. A urine culture was then requested, which had no growth.

The patient was seen by a urologist, who performed cystoscopy the following day. The urologist found the left ureteral orifice and intermural ureter were inflamed and swollen with a pinhole at the ureteral orifice. The most distal ureter had a 1$\mathrm{cm}$ length of fibrosis. The urologist dilated this area and placed a stent.

The patient later returned to the urologist, who removed the stent, and she has done well since. A follow-up imaging study has not been recommended. She will continue care with her family physician.

\section{Discussion}

The patient complained of abdominal pain and reported a history of diverticulitis. Although she was afebrile, she did have leukocytosis. Findings on her abdominal examination were suggestive of diverticulitis. The costovertebral angle tenderness was explained by presumed irritation of the posterior peritoneum. She had no history of renal problems, including nephrolithiasis, and her urinalysis results were unremarkable. Her pain was not colicky, and the paucity of red cells in her urine led us to believe that a renal or urologic cause was unlikely.

Details of the patient's earlier episode of diverticulitis were obtained and found to be similar to those of this episode. She also described her history of cervical cancer and radium treatment, but that information was used only to consider partial bowel obstruction. Nonocclusive mesenteric infarction was in the differential diagnosis, considering the patient's history of vascular disease and her failure to respond to antibiotic therapy; nevertheless, the pain was not exacerbated by eating, and the patient had a normal anion gap. Although the patient's symptoms and signs were somewhat unusual for diverticulitis, they were more unusual for the other conditions included in the differential diagnosis.

The patient's presumed diverticulitis was treated on an outpatient basis. When she returned after having worsened, she underwent additional tests and was then hospitalized for inpatient treatment of diverticulitis. When she failed to improve as expected, further diagnostic evaluation was obtained, including consultation and computed tomograms. A revised diagnosis was made, and she was given appropriate treatment. Rather than making the diagnosis of an unusual case of diverticulitis, we could have first expanded our differential diagnosis. In retrospect, I believe that this patient was managed properly. I suspect that her previous diverticulitis was probably an episode of ureter rupture that healed spontaneously.

Ureteral stenosis caused by radiotherapy for cervical cancer has an actuarial increase in incidence of 0.15 percent per year, and an incidence of 2.5 percent at 20 years, with the latest diagnosis of ureter stricture made 29 years after treatment. ${ }^{1}$ In another study, ${ }^{4} 10$ of 1226 patients ( 0.8 percent) treated with radium implants for cervical cancer developed ureter strictures. Two types of strictures were described, one of which was a "localized stricture of the distal ureter caused by irradiation fibrosis in the parametrial tissues," the same condition found in the patient described above. Other documented prevalences of stricture as a result of radiotherapy are 5 percent ${ }^{5}$ and 1.8 percent. ${ }^{6}$ In none of these studies was ureter rupture listed as a complication.

The clinical picture of this patient was not unusual for patients with a ruptured ureter. In one series of 27 patients with ureter rupture ${ }^{13}$ (all from nephrolithiasis), all patients had sudden onset of symptoms; violent, deep, constant pain; and rigidity of the corresponding hemiabdomen. Also in this series 23 patients had flank tenderness, and 12 had rigidity with rebound tenderness of the corresponding hemiabdomen. That the urine was not 
more remarkable is of interest. In many cases of ureter rupture, hematuria is absent. One study found 5 of 16 patients ( 31 percent), ${ }^{11}$ another 7 of 24 patients ( 29 percent), ${ }^{13}$ and a third 19 of 51 patients $\left(37\right.$ percent) ${ }^{15}$ with ureteral injury had no hematuria.

Based on the cystoscopy findings, the patient did have fibrosis and stricture of the distal ureter. Obstruction was manifested by hydronephrosis. The ureter was probably weakened and then ruptured from the increased pressure. This phenomenon of increased pressure leading to rupture has been described with associated urolithiasis, ${ }^{14}$ but there was no evidence of urolithiasis in this case. Most likely some noncalculous urinary debris became lodged in a stenotic portion of the ureter.

\section{Summary}

Ureter stricture is a not uncommon complication of radium treatment for cervical cancer, but up to now ureter stricture has not been described to result in ureter rupture. Although ureter rupture can occur for many reasons, this case is the first reported of rupture occurring as a result of treatment with radium implants. This case should encourage the clinician to add ureter rupture to the differential diagnosis of an acute abdomen and to consider ureter problems in women with a history, however distant, of radiotherapy; even with an absence of hematuria.

This case also highlights the care of a patient with an unusual constellation of symptoms and signs for which the diagnosis is uncertain. As long as a patient remains stable, proceeding with appropriate treatment for the presumed diagnosis is reasonable; however, rethinking the diagnosis if the patient fails to improve is appropriate. The physician's clinical judgment determines the extent and timing of diagnostic evaluation.

\section{References}

1. McIntyre JF, Eifel PJ, Levenback C, Oswald MJ. Ureteral stricture as a late complication of radiotherapy for stage $1 \mathrm{~B}$ carcinoma of the uterine cervix. Cancer 1995;75:836-43.

2. Hartenbach EM, Saltzman AK, Carter JR, Fowler JM, Hunter DW, Carlson JW, et al. Nonsurgical management strategies for the functional complications of ileocolonic continent urinary reservoirs. Gynecol Oncol 1995;59:358-63.

3. Schneider AW, Conrad S, Busch R, Otto U. The cold-knife technique for endourological management of stenoses in the upper urinary tract. J Urol 1991;146:961-5.

4. Shingleton HM, Fowler WC Jr, Pepper FD Jr, Palumbo L. Ureteral strictures following therapy for carcinoma of the cervix. Cancer 1969;24:77-83.

5. Underwood PB, Lutz MH, Smoak DL. Ureteral injury following irradiation therapy for carcinoma of the cervix. Obstet Gynecol 1977;49:663-9.

6. Slater JM, Fletcher GH. Ureteral strictures after radiation therapy for carcinoma of the uterine cervix. Am J Roentgenol Radium Ther Nucl Med 1971;111: 269-72.

7. Francesca F, Scattoni V, Nava L, Pompa P, Grasso M, Rigatti P. Failures and complications of transurethral ureteroscopy in 297 cases: conventional rigid instruments vs. small caliber semirigid ureteroscopes. Eur Urol 1995;28:112-5.

8. Meyer NL, Lipscomb GH, Ling FW. Ureteral injury during pregnancy termination. A case report. J Reprod Med 1994;39:743-6.

9. Hall SJ, Carpinito GA. Traumatic rupture of a renal pelvis obstructed at the ureteropelvic junction: case report. J Trauma 1994;37:850-2.

10. Reznichek RC, Brosman SA, Rhodes DB. Ureteral avulsion from blunt trauma. J Urol 1973;109:812-6.

11. Presti JC Jr, Carroll PR, McAninch JW. Ureteral and renal pelvic injuries from external trauma: diagnosis and management. J Trauma 1989;29:370-4.

12. Ginsberg SA. Spontaneous urinary extravasation in association with renal colic. J Urol 1965;94:1925.

13. Orkin LA. Spontaneous or nontraumatic extravasation from the ureter. J Urol 1952;67:272-83.

14. Schwartz A, Caine $M$, Hermann G, Bittermann $W$. Spontaneous renal extravasation during intravenous urography. Am J Roentgenol Radium Ther Nucl Med 1966;98:27-40.

15. Bright TC, Peters PC. Ureteral injuries due to external violence: 10 years' experience with 59 cases. J Trauma 1977;17:616-20. 